\title{
CUGC for hyperornithinemia-hyperammonemia-homocitrullinuria (HHH) syndrome
}

\author{
Diego Martinelli $\mathbb{D}^{1}$ - Giuseppe Fiermonte ${ }^{2}$ - Johannes Häberle ${ }^{3}$. Sara Boenzi ${ }^{1}$ - Bianca Maria Goffredo ${ }^{1}$. \\ Lorena Travaglini $^{4} \cdot$ Emanuele Agolini $\mathbb{1}^{5} \cdot$ Vito Porcelli $^{2} \cdot$ Carlo Dionisi-Vici $^{1}$
}

Received: 2 May 2018 / Revised: 3 March 2020 / Accepted: 10 March 2020 / Published online: 2 April 2020

(c) European Society of Human Genetics 2020

\section{Disease characteristics}

\subsection{Name of the disease (synonyms)}

Hyperornithinemia-hyperammonemia-homocitrullinuria (HHH) syndrome.

\subsection{OMIM\# of the disease}

238970.

\subsection{Name of the analysed genes or DNA/ chromosome segments}

SLC25A15 or ORNT1 at 13q14.11.

Supplementary information The online version of this article (https:// doi.org/10.1038/s41431-020-0616-x) contains supplementary material, which is available to authorized users.

Diego Martinelli

diego.martinelli@opbg.net

$\triangle$ Carlo Dionisi-Vici

carlo.dionisivici@opbg.net

1 Division of Metabolism and Research Unit of Metabolic Biochemistry, Bambino Gesù Children's Hospital, IRCCS, Rome, Italy

2 Department of Biosciences, Biotechnologies and Biopharmaceutics, University of Bari, Bari, Italy

3 Division of Metabolism and Children's Research Center, University Children's Hospital Zurich, Zurich, Switzerland

4 Unit of Neuromuscular and Neurodegenerative Disorders, Laboratory of Molecular Medicine, Bambino Gesù Children's Hospital, IRCCS, Rome, Italy

5 Molecular Genetics Laboratory, Bambino Gesù Children's Hospital, IRCCS, Rome, Italy

\subsection{OMIM\# of the gene(s)}

603861.

\subsection{Mutational spectrum}

From 1999 to date, 50 affecting function variants have been identified and associated to HHH syndrome [1-5]. As it is not available in the literature a complete up-to-date list of disease-causing variants for SLC25A15 gene, we included this information as a Supplementary Excel sheet (See Supplementary Material File \#1): this list was created by using LOVD and ClinVar databases and liked to the relevant literature reference. Reported variants consist of: 29 missense variants, 4 frameshift, 11 nonsense, 2 splicing, 2 small deletion, 1 in frame insertion, 1 gross deletion.

\subsection{Analytical methods}

Sanger sequencing of coding regions and flanking intronic sequences of SLC25A15 gene is the mainstay analytical method (NCBI reference sequence: NG_012248.1).

\subsection{Analytical validation}

There are several steps in the analytical validation process.

- Sequencing of both DNA strands (forward and reverse) is performed. Although most of variants have been identified by using this approach, intronic variants, large deletions, and duplications may require a different methodology.

- It is recommended to check the identified heterozygous or homozygous variants also in patients' parents. Proof of segregation further supports the diagnosis.

- Pathogenicity of variants can be investigated by using an in vitro yeast cell model [6] and by reconstitution of the bacterial-expressed recombinant mutant transporters into artificial phospholipids vesicles [7]. 


\subsection{Estimated frequency of the disease (incidence at birth ("birth prevalence") or population prevalence. If known to be variable between ethnic groups, please report)}

Up to date, around 122 affected patients have been reported [2-4]. In a recent review of 456 Urea Cycle Disorders (UCDs) patients prospectively followed between 2011 and 2015 by the European Registry and Network for Intoxication type Metabolic Diseases (E-IMD: https://www.e-imd) patient registry, $11 \mathrm{HHH}$ syndrome patients were reported; thus, this disorder accounted for $2.4 \%$ of all UCDs subjects [8]. Although the HHH syndrome can be considered a very rare disease with a panethnic distribution, it has been more frequently reported in three countries: 26 patients (22\%) were Canadian, as a result of a founder variant in Quebec [1], 18 patients (15\%) were Italian, and 14 patients (12\%) were Japanese. In these three countries the HHH syndrome should not be considered a very rare disease since they account for about the $50 \%$ of all reported patients.

\subsection{Diagnostic setting}

\begin{tabular}{lll}
\hline & Yes. & No. \\
A. (Differential) diagnostics & $\bigotimes$ & $\square$ \\
B. Predictive Testing & $\bigotimes$ & $\square$ \\
C. Risk assessment in Relatives & $\bigotimes$ & $\square$ \\
D. Prenatal & $\bigotimes$ & $\square$ \\
\hline
\end{tabular}

Comment: HHH syndrome is a rare recessive UCD [9] caused by variants in the $S L C 25 A 15$ gene $[1,2,5]$, which encodes for the mitochondrial ornithine transporter (ORNT1), a member of the mitochondrial carrier family which, by exchanging cytosolic ornithine with matrix citrulline across the inner mitochondrial membrane, connects the cytosolic and the intramitochondrial reactions of the urea cycle $[1,6]$. Disease onset usually ranges from early infancy to childhood and, in rarer cases, adulthood. Patients typically present a variable clinical spectrum, ranging from a mild form with aversion for protein-rich foods, developmental delay/intellectual disability, myoclonic seizures, ataxia and pyramidal dysfunction, to a more severe acute form with intermittent episodes of vomiting, confusion or coma, acute liver failure or hepatitis-like attacks [3]. The chronic course of $\mathrm{HHH}$ syndrome is characterized by progressive encephalopathy with mental regression, and early signs of motor neuron dysfunction (spastic paraparesis) [2, 3]. Multiple supratentorial stroke-like lesions have also been reported, while ophthalmological signs are rare, with few patients showing severe retinal degeneration $[3,10]$. The late onset cases present with hyperammonemia accompanied by lethargy and/or coma and signs of severe liver dysfunction and/or life-threatening episodes of acute liver failure [3].

Asides from the severe neonatal form, there is no evidence of a direct correlation between age of onset, which is variable, and disease severity. No clear phenotype-genotype correlations could be found. Early diagnosis in infancy or childhood may improve the clinical outcome. The diagnosis relies on clinical signs and the peculiar metabolic triad of hyperammonemia, hyperornithinemia, and urinary excretion of homocitrulline, the latter probably generated due to the lower matrix levels of ornithine which force ornithine transcarbamylase to use lysine as alternative substrate instead of ornithine. Besides this biochemical signature, patients may also present with increased plasma glutamine levels and intermittent elevation of urinary orotic acid [3]. Some subjects also demonstrate low plasma creatine, as increased ornithine levels in this disorder inhibit arginine:glycine amidinotransferase, the first step of creatine synthesis [10]. Furthermore, secondary creatine deficiency may be due to low cellular arginine availability [11]. HHH syndrome enters in the differential diagnosis with other UCDs [9].

Parents and relatives of HHH syndrome affected probands should be checked for the presence of the identified variant(s). If a family has an affected child and wishes to have more children, prenatal diagnosis should be discussed in detail during genetic counseling.

\section{Test characteristics}

\begin{tabular}{|c|c|}
\hline $\begin{array}{l}\text { Genotype or } \\
\text { disease }\end{array}$ & $\begin{array}{l}\text { A: True } \\
\text { positives }\end{array}$ \\
\hline Present Absent & $\begin{array}{l}\text { B: False } \\
\text { positives }\end{array}$ \\
\hline
\end{tabular}

Test

$\begin{array}{llll}\text { Pos. A } & \text { B } & \text { Sensitivity: } & \mathrm{A} /(\mathrm{A}+\mathrm{C}) \\ & & \text { Specificity: } & \mathrm{D} /(\mathrm{D}+\mathrm{B}) \\ \text { Neg. C } & \text { D } & \text { Pos. } & \mathrm{A} /(\mathrm{A}+\mathrm{B}) \\ & & \text { predict. value: } & \mathrm{D} /(\mathrm{C}+\mathrm{D}) \\ & & \\ & \text { Neg. } & \\ & \text { predict. value: } & \\ & & \end{array}$

\subsection{Analytical sensitivity}

(proportion of positive tests if the genotype is present)

$100 \%$, if the genotype is also associated to at least one out of the three metabolic hallmarks, hyperammonemia, hyperornithinemia, and homocitrullinuria. 


\subsection{Analytical specificity}

(proportion of negative tests if the genotype is not present)

Nearly $100 \%$, if the genotype is also associated to the absence of hyperammonemia, hyperornithinemia, and homocitrullinuria.

\subsection{Clinical sensitivity}

(proportion of positive tests if the disease is present)

The clinical sensitivity can be dependent on variable factors such as age or family history. In such cases a general statement should be given, even if a quantification can only be made case by case.

When the diagnosis has been properly established based on clinical and genetic investigations, family history and biochemical results, very few negative tests are expected. When genetic testing is negative in a patient presenting only one or two out of the three metabolic hallmarks, hyperammonemia, hyperornithinemia, and homocitrullinuria, a differential diagnoses should be considered. Notably, some patients may show an incomplete biochemical phenotype and $\mathrm{HHH}$ syndrome presents with a lower degree of hyperammonemia compared with other UCDs [3, 9]. These cases can likely result in a lower sensitivity.

\subsection{Clinical specificity}

(proportion of negative tests if the disease is not present)

The clinical specificity can be dependent on variable factors such as age or family history. In such cases a general statement should be given, even if a quantification can only be made case by case.

Close to $100 \%$. A precise quantification is difficult, since molecular testing of SLC25A15 gene is not performed on a routine basis in asymptomatic individuals.

\subsection{Positive clinical predictive value}

(life time risk to develop the disease if the test is positive)

The positive clinical predictive value is $100 \%$. Although the onset of symptoms is in most cases in infancy and childhood, the diagnosis is often delayed, with at least one fourth of cases identified in adulthood [3]. Furthermore, a few patients presented the first disease manifestation as adults. As already described, genetic analysis does not have a prognostic value, since even in the same family and with the same variant, the phenotype can be quite variable [3].

\subsection{Negative clinical predictive value}

(probability not to develop the disease if the test is negative)

Assume an increased risk based on family history for a non-affected person. Allelic and locus heterogeneity may need to be considered.

Index case in that family had been tested:

The negative clinical predictive value is nearly $100 \%$.

Index case in that family had not been tested:

Genetic testing for a clinically unaffected individual is not indicated in this situation. It would only be undertaken if a variant in the $S L C 25 A 15$ gene has been identified in the proband.

\section{Clinical utility}

\section{1 (Differential) diagnostics: the tested person is clinically affected}

(To be answered if in 1.9 "A" was marked)

\subsubsection{Can a diagnosis be made other than through a} genetic test?
No.

Yes.

\subsubsection{Describe the burden of alternative diagnostic methods to the patient}

The blood and urine samplings for the serum ammonia and ornithine and urine homocitrulline (and orotic acid) measurements is a minor burden to the patient. The blood sampling is also used for the genetic analysis which helps to confirm the clinical diagnosis.

\subsubsection{How is the cost effectiveness of alternative diagnostic methods to be judged?}

In some countries, like United States and Canada, $\mathrm{HHH}$ syndrome is included in the disease panel of expanded 
newborn screening programs. In others, where this diseases is not screened at birth, like in Italy, the costs of biochemical and genetic tests in clinically symptomatic patients are covered by National Health systems. In general, since SLC25A15 gene is small and thus accessible to Sanger sequencing, costs of genetic testing are low in comparison with a too late diagnosis during a metabolic crisis requiring intensive care treatment.

\subsubsection{Will disease management be influenced by the result of a genetic test?}

No.

Yes. $\triangle$

Therapy (please An appropriate management with describe) (continued)

Prognosis (please
describe)

avoid secondary creatine deficiency $[3,11]$. describe)

Prognosis is highly variable ranging from mild neurological involvement to a severely disabling disease. Seven patients out of the published 122 patients, with a known vital status, died. Despite dietary and pharmacological treatment, most of patients reaching adult age present with variable degree of neurological and cognitive impairment and psychiatric symptoms (such as abnormal gait, ataxia, episodic confusion, disorientation, incoherence, agitation, restlessness, headache) [12]. Successful pregnancies have been reported in $\mathrm{HHH}$ syndrome female patients $[3,4]$.

Management Since HHH syndrome can affect (please describe) different systems, follow-up by a multidisciplinary team is crucial. In particular, as the evolution of spastic paraparesis is unaffected by pharmacological treatment, regular physical and occupational therapy as well as drugs able to reduce spasticity are recommended; periodical clinical and neurophysiological evaluation of the progression of the disease is advisable to direct rehabilitation as in other inherited spastic parapaplegias [13]. In particular, neurophysiological studies highlight the progressive degeneration of the corticospinal tracts in $\mathrm{HHH}$ syndrome, clearly predominant if compared to a less frequent involvement of central sensory system and peripheral nervous system [14]. These data give insight on the pathogenesis of the disease and can help in directing rehabilitation approaches [14]. The metabolic follow-up includes measurement of plasma ammonia, amino acids (and possibly creatine), urinary homocitrulline and orotic acid. 
3.2 Predictive Setting: The tested person is clinically unaffected but carries an increased risk based on family history

(To be answered if in 1.9 "B" was marked)

3.2.1 Will the result of a genetic test influence lifestyle and prevention?

If the test result is positive (please describe):

Although an appropriate and chronic therapy (see 3.1.4) prevents hyperammonemia and liver disease, it does not prevent the spastic paraparesis progression. If positive, the test will greatly influence the choice of career and life planning.

If the test result is negative (please describe):

If the variant is identified in the index case and not in an unaffected proband, regular examinations are not necessary. Follow-up is recommended only if the disease-causing variant could not be identified.

\subsubsection{Which options in view of lifestyle and prevention does} a person at-risk have if no genetic test has been done (please describe)?

That person at-risk should avoid a protein-enriched diet in order to prevent life-threatening hyperammonemic crisis.

\subsection{Genetic risk assessment in family members of a diseased person}

(To be answered if in 1.9 "C" was marked)

\subsubsection{Does the result of a genetic test resolve the genetic situation in that family?}

Yes, a molecular diagnosis in an affected individual can resolve the genetic situation in that family. A positive test in a patient may lead, at adult age, to test the carriership of his/ her partner.

\subsubsection{Can a genetic test in the index patient save genetic or} other tests in family members?

No, if a disease-causing variant is identified in the index patient, family members should be tested in order to avoid a possible variant's spreading in the adult age.

\subsubsection{Does a positive genetic test result in the index patient} enable a predictive test in a family member?

If a disease-causing variant is identified in the index patient, it may help to quickly identify another affected relative since very often affected patients show an aversion to protein-rich foods.

\subsection{Prenatal diagnosis}

(To be answered if in 1.9 " $\mathrm{D}$ " was marked)

3.4.1 Does a positive genetic test result in the index patient enable a prenatal diagnosis?

Yes. Prenatal diagnosis should be performed by molecular analysis.

\section{If applicable, further consequences of testing}

Please assume that the result of a genetic test has no immediate medical consequences. Is there any evidence that a genetic test is nevertheless useful for the patient or his/her relatives? (Please describe)

Genetic testing for SLC25A15 variants will provide a molecular diagnosis, allowing an appropriate management of the patient, reducing the risk of hyperammonemia and metabolic decompensation. It will also define the inheritance patterns and enable effective genetic counseling.

Acknowledgements This work was supported by EuroGentest2 (Unit 2: "Genetic testing as part of health care"), a Coordination Action under FP7 (Grant Agreement Number 261469) and the European Society of Human Genetics.

\section{Compliance with ethical standards}

Conflict of interest The authors declare that they have no conflict of interest.

Publisher's note Springer Nature remains neutral with regard to jurisdictional claims in published maps and institutional affiliations.

\section{References}

1. Camacho JA, Obie C, Biery B, Goodman BK, Hu CA, Almashanu $\mathrm{S}$, et al. Hyperornithinaemia-hyperammonaemia-homocitrullinuria syndrome is caused by mutations in a gene encoding a mitochondrial ornithine transporter. Nat Genet. 1999;22:151-8.

2. Debray FG, Lambert M, Lemieux B, Soucy JF, Drouin R, Fenyves D, et al. Phenotypic variability among patients with hyperornithinaemia-hyperammonaemia-homocitrullinuria syndrome homozygous for the delF188 mutation in SLC25A15. J Med Genet. 2008;45:759-64.

3. Martinelli D, Diodato D, Ponzi E, Monné M, Boenzi S, Bertini E, et al. The hyperornithinemia-hyperammonemia-homocitrullinuria syndrome. Orphanet J Rare Dis. 2015;10:29.

4. Camacho J, Rioseco-Camacho N. Hyperornithinemia-Hyperammonemia-Homocitrullinuria Syndrome. In: Adam MP, Ardinger HH, Pagon RA, Wallace SE, Bean LJH, Stephens K, 
Amemiya A, editors. GeneReviews ${ }^{\circledR}$. Seattle (WA): University of Washington; 1993-2020. 2012.

5. Tessa A, Fiermonte G, Dionisi-Vici C, Paradies E, Baumgartner MR, Chien Y-H, et al. Identification of novel mutations in the SLC25A15 gene in hyperornithinemia-hyperammonemiahomocitrullinuria $(\mathrm{HHH})$ syndrome: a clinical, molecular, and functional study. Hum Mutat. 2009;30:741-8.

6. Marobbio CMT, Punzi G, Pierri CL, Palmieri L, Calvello R, Panaro MA, et al. Pathogenic potential of SLC25A15 mutations assessed by transport assays and complementation of Saccharomyces cerevisiae ORT1 null mutant. Mol Genet Metab. 2015;115:27-32.

7. Fiermonte G, Dolce V, David L, Santorelli FM, Dionisi-Vici C, Palmieri $\mathrm{F}$, et al. The mitochondrial ornithine transporter. Bacterial expression, reconstitution, functional characterization, and tissue distribution of two human isoforms. J Biol Chem. 2003;278:32778-83.

8. Posset R, Garcia-Cazorla A, Valayannopoulos V, Teles EL, Dionisi-Vici C, Brassier A, et al. Age at disease onset and peak ammonium level rather than interventional variables predict the neurological outcome in urea cycle disorders. J Inherit Metab Dis. 2016;39:661-72.

9. Häberle J, Boddaert N, Burlina A, Chakrapani A, Dixon M, Huemer M, et al. Suggested guidelines for the diagnosis and management of urea cycle disorders. Orphanet J Rare Dis. 2012;7:32.

10. Morini C, Capozzi P, Boenzi S, Rizzo C, Santorelli FM, DionisiVici C. Retinal degeneration. Ophthalmology. 2009;116:1593.

11. Boenzi S, Pastore A, Martinelli D, Goffredo BM, Boiani A, Rizzo $\mathrm{C}$, et al. Creatine metabolism in urea cycle defects. J Inherit Metab Dis. 2012;35:647-53.

12. Kim SZ, Song WJ, Nyhan WL, Ficicioglu C, Mandell R, Shih VE. Long-term follow-up of four patients affected by HHH syndrome. Clin Chim Acta. 2012;413:1151-5.

13. Blackstone C. Hereditary spastic paraplegia. Handb Clin Neurol. 2018;148:633-52.

14. Olivieri G, Pro S, Diodato D, Di Capua M, Longo D, Martinelli D, et al. Corticospinal tract damage in HHH syndrome: a metabolic cause of hereditary spastic paraplegia. Orphanet J Rare Dis 2019;14:208. 